\title{
LA COUR SUPREME ET LE PARTAGE DES COMPETENCES LEGISLATIVES
}

\author{
GÉRALD A. BEAUDOIN*
}

\begin{abstract}
L'auteur du présent article a lu le texte de la communication du professeur JeanCharles Bonefant retenu d Québec et ajoute ses observations personnelles sur la Cour Suprême et le partage des compétences législatives. Il souligne les arrêts marquants des dernières décennies. Il est évident que dans le domaine du partage des compétences et des droits fondamentaux la Cour Suprême joue un róle considérable et irremplacable. C'est la sa plus haute mission.

The author of the following article has read the text of Professor Jean-Charles Bonefant on the Supreme Court and the distribution of legislative powers and has added his own personal observations. He analyses succinctly some of the most important cases in the last decades. In the area of the legislative powers and fundamental freedoms, our highest tribunal plays a role that no other institution may do in its stead and place. This is the most important and noble task assigned to the Court.
\end{abstract}

G. A. B.

A la suite de l'exposé du Professeur Jean-Charles Bonefant, on me permettra d'ajouter quelques notes sur la Cour Suprême et le partage des compétences législatives.

Au chapitre du pouvoir de dépense, je formule l'espoir que notre Cour Suprême soit appelée à se prononcer. L'exercice de cette compétence fédérale dans des domaines autres que la préréquation et la lutte aux disparités régionales soulève de l'anxiété au Québec. Une décision de 1937 du Comité Judiciaire du Conseil Privé porte sur l'existence et l'exercice de cette compétence mais au fond elle ne constitue qu'un "caveat". ${ }^{1}$ La Cour de l'Echiquier ${ }^{2}$ à quelques reprises a rendu des décisions en la matière. Il est à souhaiter que ce pouvoir soit délimité par notre plus haut tribunal. Dans un état fédératif le pouvoir de taxer et de dépenser joue un rôle cardinal. ${ }^{3}$ Je me surprends que ce pouvoir n'ait pas même fait l'objet d'une opinion consultative ou d'un jugement, de la part de la Cour Suprême.

Sur le plan des droits linguistiques, la Cour Suprême a adopté une attitude remarquable dans l'arrêt Jones ${ }^{4}$ et je me plais à l'en féliciter! Le jugement unanime de la Cour, rendu sous la plume du nouveau Juge en Chef reconnaît le droit au législateur central, principalement par le jeu de sa compétence résiduelle, d'aller au-delà de l'article 133 sans le contredire et de promouvoir le bilinguisme dans les institutions qui relèvent du Gouvernement fédéral et du Parlement.

Qu'arriverait-il si la loi québécoise sur la langue officielle ${ }^{5}$ adoptée en juillet 1974 devait faire l'objet d'un examen par la Cour Suprême? J'ai déjà eu l'occasion d'ćtudier la question et de conclure que la Cour Suprême aurait bien des chances de déclarer cette loi constitutionelle dans son ensemble. ${ }^{\circ}$ Il se peut que cette mesure contienne ici et là des accrocs à la Constitution, mais ils ne m'apparaissent pas majeurs et peuvent être isolés. La constitutionnalité de la mesure considérée dans son ensemble ne m’apparaît pas douteuse.

- Dean, Université D'Ottawa, Faculté De Droit.

1 A.G. for Canada v. A.G. for Ontario, [1937] A.C. 326.

2 Angers v. The Minister of National Revenue, [1957] R.C. de l'E. 83; Porter v. La Reine, [1965] 1 R.C. de l'E. 200.

3 P. E. Trudeau, Le fédéralisme et la société canadienne française, à 145.

4 Jones v. A.G. of Canada, [1974] 16 C.C.C. (2d) 297.

5 L.Q. 1974, c. 6 (sanctionnée le 31 juillet 1974).

6 G. A. Beaudoin, La loi 22 et la Constitution, (1974) 5 (1) R.G.D. 169. 
Dans le domaine du pouvoir déclaratoire, ${ }^{7}$ il est possible que la Cour Suprême et surtout le Comité Judiciaire du Conseil Privé se soient montrés trop généreux. Les cours auraient avantage à circonscrire le mot "works". Il faut restreindre le pouvoir déclaratoire qui constitue un accroc sérieux aux principes de base du fédéralisme. Dans l'arrêt Eastern Terminal Elevators, ${ }^{8}$ le savant Juge Duff de la Cour Suprême avait suggéré que le Parlement utilise ce pouvoir pour régler le problème du commerce du blé. Cette suggestion était bien fondée. Cependant le Parlement ne fut pas aussi bien inspiré lorsqu'il recourut à ce pouvoir pour des travaux qui ne sont à l'avantage du Canada que de façon fort éloignée. ${ }^{9}$ Cependant, le premier grief s'adresse plutôt aux auteurs de la Constitution ou au Parlement. La Cour - on doit le reconnaître - se trouve dans une situation difficile. Elle se prononce sur un choix arrêté dans l'arène politique.

Au plan des télécommunications et de la télévision, il existe, comme on le sait, un contentieux entre Quèbec et Ottawa. La câblovision et la télévision éducative n'ont pas été considérées par le Comité judiciaire du Conseil Privé en 1932. ${ }^{10}$ Des cours provinciales et la Cour fédérale d'Appel ont déclaré que le contenant et le contenu des ondes relèvent exclusivement de l'autorité centrale. ${ }^{11}$ Il est à souhaiter que la Cour Suprême ait à trancher ce débat. Ce sera certes un arrêt majeur en matière de partage des compétences législatives.

En matière d'administration de la justice, la Cour Suprême a rendu des arrêts marquants sur la portée de l'article 96 . Le renvoi sur la loi ontarienne en matière d'adoption ${ }^{12}$ constitue une anlyse appropriée de la portée de l'article 96 de la Constitution. L'attitude prise par la Cour Suprême sur ce point dans le renvoi sur la constitutionnalité de la loi sur la Cour de Magistrat ${ }^{13}$ et dans l'affaire

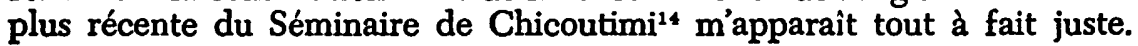

Au titre des richesses naturelles, la décision de 1967 sur les droits miniers sous-marins ${ }^{15}$ n'a pas fini d'attirer l'attention des juristes. Des critiques ont été formulées tant sur le plan de la décision elle-même que sur la formulation des motifs. ${ }^{18}$

7 British North America Act, 1867, 30-31 Vict., c. 3, art. 92 (10) (Imp.).

${ }^{8}$ R. v. Eastern Terminal Elevator Co. [1925] R.C.S. 434.

- Voir sur ce plan A. Lajoie, Le pouvoir déclaratoire du Parlement, à 54.

10 In re Regulation and Control of Radio Communication in Canada, [1932] A.C. 304.

11 Sur la cablovision, Re Public Utilities Commission and Victoria Cablevision Ltd., (1965) 5 l D.L.R. (2d) 716; R. v. City of New Westminster, ex parte Canadian Wirevision Ltd., (1966) 55 D.L.R. (2d) 613.

12 In the matter of a reference concerning the authority of judges and junior and acting judges of the county and district courts, police magistrates, justices of the peace and judges of juvenile courts, to perform the functions vested in them respectively by the legislature of the Province of Ontario pursuant to the provisions of the Adoption Act; thie Children's Protection Act; the Children of Unmarried Parents Act; and the Deserted Wives' and Children's Maintenance Act; being chapters 218, 312, 217 and 211 respectively of the Revised Statutes of Ontario, 1937, [1938] R.C.S. 398.

18 Renvoi touchant la constitutionnalité de la loi concernant la juridiction de la Cour de Magistrat, [1965] R.C.S. 772.

14 Séminaire de Chicoutimi et le Proc. Gen. et Ministre de la Justice de la Province de Québec v. La Cité de Chicoutimi, [1973] R.C.S. 681.

${ }^{15}$ In the matter of a reference by the Governor General in Council concerning the ownership of and jurisdiction over offshore mineral rights as set out in order in councll P.C. 1965-750,dated April 26, 1965, [1967] R.C.S. 792.

${ }^{16} \mathrm{~J}$. Brossard, article paru dans Le Devoir du 22 novembre 1967 à 5; I. Head, The Canadian Offshore Minerals Reference, (1968) U. of T.L.J. 131; E. McWhinney, Canadian Federalism and the Foreign Affairs and Treaty Power: the Impact of Quebec's Quiet Revolution, (1969) 7 A.C.D.I. 3; A. Dufour, Fédéralisme canadien et droit international, dans Macdonald, Morris et Johnston (éds.), Canadian Perspective on International Law and Organization, à 72. 
Disons un mot de la théorie dite des “dimensions nationales" dont les contours sont imprécis et menacent la nécessaire autonomie des provinces. On admet au Québec la théorie du l'urgence nationale mais on se méfie de la théorie dite des "dimensions nationales". ${ }^{17}$ Sur ce point, la Cour Suprême serait peutêtre bien inspirée de ne pas asseoir ces décisions sur ce seul point d'appui: ce n'est pas parce qu'un problème local prend de l'ampleur qu'il doive nécessairement sortir de la sphère législative provinciale. L'équilibre délicat entre les pouvoirs que suppose le fédéralisme s'accomode mal de ce critère mal défini qui menace de trop favoriser un pouvoir au dépens de l'autre. Quand il s'agit d'un domaine qui entre dans le résiduum des compétences, il ne se pose pas de problème constitutionnel. Cependant, si nous sommes en présence d'un problème régional qui a atteint des dimensions dites nationales, nous nous trouvons donc dans une situation beaucoup plus délicate. En pareil cas, la Cour Suprême oriente nettement le fédéralisme. Il lui faut alors user de beaucoup de prudence.

Relativement aux droits fondamentaux, il faut féliciter la Cour Suprême pour les excellentes décisions qu'elle a rendues entre 1950 et $1960 .{ }^{18}$ Les résultats dans l'ensemble sont remarquables si l'on excepte un ou deux cas comme l'arrêt Birks par exemple. ${ }^{19}$ Il y a malheureusement assez d'ambuiguité dans la formulation des motifs. ${ }^{20}$ Pour ce qui est de la protection des droits fondamentaux depuis l'avènement de la Déclaration canadienne des droits, la Cour Suprême a fait preuve de beaucoup de réserve si l'on excepte l'arrêt Drybones. ${ }^{21}$ Nous entrons dans une période d'incertitude. On peut se demander, d'ailleurs, si la théorie du stare decisis continue de s'appliquer. En effet, M. le Juge Ritchie majoritaire dans l'affaire Drybones et majoritaire dans l'affaire Lavell ${ }^{22}$ s'appuie sur un passage de M. le Juge Pigeon qui lui était dissident dans l'affaire Drybones. Pour la protection des droits fondamentaux, il y a deux écoles de pensée. Il m'apparaît que Messieurs les Juges Pigeon et Laskin, qui représentent bien les deux écoles, sont constants et logigues avec eux-mêmes. Le premier se refuse à voir dans cette déclaration plus qu'une loi d'interprétation, alors que le second y voit une mesure quasi-constitutionnelle qui justifie un "judicial review" de la part de la Cour, à moins que le Parlement n'ait déclaré que sa mesure s'appliquait nonobstant la Déclaration de 1960.

Dans le domaine du pouvoir d'urgence, la Cour Suprême, dans l'affaire de la Régie des loyers, a confirmé l'attitude prise par le Conseil Privé dans l'arrêt Fort Frances. Ce pouvoir est implicite dans la Constitution. Le Juge Rand toutefois se réfère au critère adopté par Lord Simon dans l’arrêt sur la tempérance de 1946.23 Au Québec les motifs de l'arrêt Fort Frances sont mieux acceptés que ceux formulés par Lord Simon dans l'arrêt de 1946.

17 Voir J. Beetz, Les attitudes changeantes du Québec d lendroit de la Constitution de 1867, dans C. B. Macpherson et P. A. Crepeau, L'Avenir du fédéralisme canadien, à 113 .

18 Boucher v. Le Roi, [1951] R.C.S. 255; Saumur v. City of Quebec, [1953] 2 R.C.S. 299; Chaput v. Romain, [1955] R.C.S. 834; Roncarelli v. Duplessis, [1959] R.C.S. 121; Lamb v. Benoit, [1959] R.C.S. 321, etc. . . .

19 Birks v. City of Montreal, [1955] R.C.S. 799.

20 Voir P. Grant, La liberté religieuse et le droit positif québecois, (1967) $27 \mathrm{R}$. du B. 357.

21 R. v. Drybones, [1970] R.C.S. 282.

22 P.G. du Canada v. Lavell, [1974] R.C.S. 1349.

${ }^{28}$ In the matter of a Reference as to the Validity of the Wartime Leasehold Regulations, P.C. 9029, [1950] R.C.S. 124; Fort Frances Pulp and Power Co. v. Manitoba Free Press Co., [1923] A.C. 695; A.G. for Ontario v. Canada Temperance Federation, [1946] A.C. 193. 
Mais en matière d'urgence, c'est surtout sur le plan législatif qu'il y a carence. Le parlement devrait édicter une loi d'urgence pour le temps de paix. Les cours sont très réticentes à intervenir en ce domaine et je n'ai pas de mal à les comprendre. Cependant, il serait avantageux que le mécanisme de l'état d'urgence soit plus clairement indiqué dans la loi fondamentale du pays afin que les cours de justice puissent intervenir en cas d'abus possible du pouvoir exécutif et du pouvoir législatif.

En matière de "mariage et de divorce", la Cour Suprême a rendu des arrêts importants ces dernières années qui m'apparaissent respecter le jeu normal des articles 91 et 92. ${ }^{24}$ Les vocables "mariage" et "divorce" qui apparaissent à l'article 91 doivent recevoir des tribunaux une interprétation. Cependant, il serait peutêtre normal que par voie d'amendement constitutionnel le mariage et le divorce reviennent aux provinces maintenant que Québec est devenu pluraliste. Québec pourrait ainsi se donner un régime de droit familial plus conforme à son génie. ${ }^{25}$ Les provinces ont déjà compétence en droit civil et en matière de célébration de mariage. Il s'agirait de s'entendre sur une définition du domicile.

Pour ce qui est de la protection des droits confessionels, je constate avec plaisir que la Cour Suprême s'est montrée plus généreuse et moins légaliste que le Comité judiciaire du Conseil Privé dans l'interprétation de l'article 93.28

Sur le plan du droit pénal l'arrêt Ross ${ }^{27}$ contribue à tirer une ligne entre les articles $91(27)$ et $92(13)$ en réaffirmant le principe que les conséquences civiles d'un acte criminel ne doivent pas être considérées comme une "peine" de façon a faire relever la question de la compétence exclusive du Parlement central. La suspension d'un permis de conduire fait en vertu de la loi provinciale est une conséquence civile. Cet arrêt est respectueux de l'autonomie des provinces en matière de propriété et de droits civils.

En matière d'interprétation des articles 91 et 92 , il faut se réjouir que la théorie du pouvoir "ancillaire" ait été appliquée aux provinces dans l'arrêt Barfried Enterprises. ${ }^{28}$

Enfin, l'arrêt Thorson ${ }^{29}$ rendu par la Cour Suprême en matière de locus standi est fort intéressant et trés bien fondé. Dans cette affaire la Cour a reconnu dans un jugement majoritaire qu'un contribuable fédéral pouvait à la discrétion de la Cour soulever l'inconstitutionnalité d'une loi fédérale. Cet arrêt qui n'a pas fait tellement de bruit en est un de très grande importance dans un état fédéral comme le nôtre. On ne semble pas avoir saisi toute la portée de cet arrêt.

L'arrêt $M c N e i l^{30}$ s'inscrit dans la même ligne que le précédent. Il applique le même principe à un contribuable provincial à l'égard d'une mesure législative provinciale. Dans cette affaire un contribuable de Nouvelle-Ecosse attaquait la constitutionnalité d'une loi de sa province et des réglements passés sous son autorité à la suite d'une décision du Bureau des censure interdisant la projection du film The Last Tango in Paris. Le Lieutenant-Gouverneur avait refusé d'accorder à ce contribuable un droit d'appel de la décision. A l'unanimité les neuf

24 Zacks v. Zacks, [1973] R.C.S. 891; Jackson v. Jackson, [1973] R.C.S. 205.

${ }_{25}$ Voir G. A. Beaudoin, De la répartition des compétences legislatives en matière de marriage et de divorce, (1970) 4 (1) R.G.D. 66.

20 Barrett v. The City of Winnipeg, [1892] 12 R.C.S. 374.

${ }^{27}$ Ross v. Régistraire des véhicules automobiles, [1975] R.C.S. 5.

${ }^{28}$ A.G. for Ontario v. Barfield Enterprise, [1963] R.C.S. 570.

29 Thorson v. P.G. du Canada, [1975] 1 R.C.S. 138.

${ }^{80}$ Arrêt McNeil, jugement rendu le 20 juin 1975. [Since rep. 5 N.R. 43 - eds.]. 
juges de la Cour Suprême reconnurent le droit du contribuable d'intenter l'action. La loi attaquée ne s’adresse pas quaux distributeurs de films et propriétaires de salles de cinéma mais au public en général.

$\mathrm{Au}$ moment où ces lignes sont écrites la Cour vient de rendre un arrêt majeur sur le contrôle par les provinces de l'acquisition de la propriété par des non résidents. Il s'agit de l'arrêt Morgan et Jacobson c. P.G. de Irle du Prince Edouard et al. . Il $^{\text {Il }}$ fut jugé qu'une législature provinciale peut réglementer lacquisition d'étendue de terres par des non résidents. Il s'agit là d'un exercice valide de la compétence provinciale en matière de propriété et droits civils. La législation sous examen n'empêche pas le non résident ou l'étranger de venir dans la province et d'y résider. La loi sous examen n'empiétait pas sur l'article 91(25). Cet arrêt m'apparait très bien fondé.

En terminant, je crois qu'il n'y a pas lieu de souhaiter lavènement d'une cour constitutionnelle pour se prononcer en matière de partage des compétences législatives. J'ai déjà eu l'occasion de traiter de ce point. ${ }^{32}$ Les causes constitutionnelles sont relativement peu nombreuses. Il est plus avantageux, je crois, que les conflits de compétence soient tranchés par la Cour générale d'Appel du pays.

La participation des provinces à la nomination des juges à la Cour Suprême, devrait être prévue dans notre Constitution. La Charte constitutionnelle canadienne de 1971 contenait une suggestion intéressante qui est un pas dans la bonne direction..$^{83}$

En terminant je formule le souhait qu'en matière de partage des compétences législatives la Cour Suprême fasse preuve d'imagination créatrice et d’audace si nécessaire et assure le fonctionnement harmonieux du jeu du fédéralisme. Il s'agit là de son rôle le plus important. Les autorités fédérale et provinciales peuvent évidemment laisser une empreinte très profonde en matière de fédéralisme. Mais en ce domaine il est un rôle que seule la Cour Suprême peut jouer. Elle a beaucoup à dire sur l'orientation du fédéralisme canadien de demain.

21 Morgan v. P.G. de l'lle-du-Prince Edouard, jugement rendu le 26 juin 1975. [Since reported 5 N.R. 455 . - eds.].

$82 \mathrm{G}$. A. Beaudoin, Cour suprême du Canada et la constitutionnalité des lois, dans Travaux des Sixièmes Journées d'Etudes Juridiques, à 187.

83 Articles 26 à 32 de la Charte constitutionnelle canadienne. 